\title{
Informative data: how to get just sufficiently rich?
}

\author{
Michel Gevers, Alex Bazanella and L. Mišković
}

\begin{abstract}
Prediction error identification requires that the selected model structure be identifiable, and that the data be informative with respect to this model structure. Whereas a range of sufficient conditions for informative experiments has been available for a long time, there were surprisingly no results of necessary and sufficient nature. With the recent surge of interest in optimal experiment design, whose solutions are typically in the form of multisines, it is of interest to know the minimal richness required of the externally applied signal to make the experiment informative. For all commonly used model structures, we provide necessary and sufficient conditions on the degree of richness of the applied signal to generate an informative experiment, both in open loop and in closed loop. In a closed-loop setup, where identification can be achieved with no external excitation if the controller is of sufficient degree, our results provide an unexpected and precisely quantifiable tradeoff between controller degree and required degree of external excitation.
\end{abstract}

\section{INTRODUCTION}

This paper takes a new look at the concept of identifiability and of informative experiments for linear time-invariant systems, both in open-loop and in closed-loop identification. Identifiability is a requirement on the chosen model structure, while the generation of data that are informative with respect to an identifiable model structure is a requirement on the experiment design. The combination of an identifiable model structure with an experiment that delivers informative data with respect to this model structure yields a well-defined identification problem, guaranteeing a unique global minimum of the identification criterion [6].

Some readers might think that everything has been said and written about these concepts, which were much studied all through the 1970's. We shared the same view ... until recently. The motivation for our renewed interest into these very fundamental questions is the recent surge of interest in the question of experiment design, itself triggered by the new concept of least costly identification experiment for robust control [2], [3], [5], [4]. In this context, questions like the following become relevant:

This work was supported by the Belgian Programme on Interuniversity Attraction Poles, initiated by the Belgian Federal Science Policy Office. The work of A. Bazanella was also partially supported by the Brazilian Ministry of Education through CAPES.

M. Gevers is with the Center for Systems Engineering and Applied Mechanics (CESAME), Université Catholique de Louvain, B-1348 Louvainla-Neuve, Belgium Michel. Gevers@uclouvain.be

A. Bazanella is with the Electrical Engineering Department, Universidade Federal do Rio Grande do Sul, Porto Alegre-RS, Brazil bazanela@ece.ufrgs.br

L. Mišković is with the Laboratory of Computational Systems Biotechnology (LCSB), Ecole Polytechnique Fédérale de Lausanne (EPFL), CH-1015 Lausanne, Switzerland. 1 jubisa.miskovic@epfl.ch
1) what is the smallest amount of external excitation that is required to generate informative data?

2) assuming that the system operates in closed-loop, when can the noise by itself generate informative data?

3 ) if, in a closed-loop experiment, noise excitation is not sufficient, then how much additional reference excitation is required?

4) assuming that excitation can be applied at different entry points of a multi-input system operating in closed loop, is it necessary to excite each input to achieve identifiability (or to achieve a given accuracy level)?

Sufficient conditions for informativity using noise excitation only (question 2) have been given, under different sets of assumptions, in [7], [8], [3]. The key condition is in terms of the complexity of the feedback controller; this complexity condition relates the controllability (or observability) indices of the controller to the controllability (or observability) indices of the plant. Question 4 has been addressed in [1] where it is shown that, when informative data cannot be generated using noise excitation only, this does not imply that all reference inputs must be excited.

In attempting to address questions 1 and 3 above, we discovered to our surprise that these questions do not seem to have been addressed (or at least solved) before. As is wellknown, besides the choice of an identifiable model structure, the key ingredient to achieve a unique global minimum of the identification criterion is the informativity of the experiment. In open-loop identification, and in all closed-loop identification experiments where the noise excitation by itself does not make the experiment informative, the informativity is achieved by applying a sufficiently rich external signal. The degree of richness of a signal is a concept that is precisely defined; a signal is said to be sufficiently rich of degree $n$ if its spectral density is nonzero in at least $n$ distinct frequency points in the interval $(-\pi, \pi]$. But whereas the scientific literature abunds with sufficient conditions on input signal richness, there appear to be no result on the smallest possible degree of richness that delivers informative data in a given identification setup. In other words, necessary conditions on input richness that will guarantee an informative experiment are strangely lacking. The recent resurgence of interest in optimal experiment design makes this question all the more relevant, because the inputs that result from optimal experiment design computations are most often expressed as multisines. It is then important to know how many different frequencies are required to ensure that these optimal inputs produce informative data.

The purpose of this contribution is to attempt to find the smallest possible degree of richness of the excitation 
signal that makes an experiment informative with respect to a chosen model structure, both in open-loop and in closedloop identification. More precisely, we address the following two questions:

- assuming open-loop identification, what is the smallest degree of input signal richness that is necessary to achieve an informative experiment with respect to a chosen model structure?

- assuming closed-loop identification with a controller that is not sufficiently complex to yield informative data using noise excitation only, what is then the smallest degree of reference signal excitation that is necessary to achieve an informative experiment with respect to a chosen model structure?

The results of this paper provide necessary and sufficient conditions for informativity of the experiment, both in open loop and in closed loop, for all commonly used singleinput single-output model structures (ARMAX, ARX, BJ and $\mathrm{OE}$ ). While the results for open-loop experiments are to be expected, our results for closed-loop experiments provide a remarkable and quantifiable trade-off between controller complexity and required degree of richness of the external excitation.

The paper is organized as follows. In Section II we set up the notations and the key tools of the prediction error identification framework. In Section III, we recall the basic concepts of identifiability and informative experiments, while in Section IV we define the degree of richness of a signal in connection with the full rank property of a corresponding regression vector. The body of our results is in Section V where we derive the minimal degree of richness that is required of the external signal to provide informative data, for all commonly utilized model structures, in both an openloop and a closed-loop setup. In line with common practice, we conclude with conclusions.

\section{THE PREDICTION ERROR IDENTIFICATION SETUP}

Consider the identification of a linear time-invariant discrete-time single-input single-output process

$$
\mathcal{S}: y(t)=G_{0}(z) u(t)+H_{0}(z) e(t)
$$

In (1) $z$ is the forward-shift operator, $G_{0}(z)$ and $H_{0}(z)$ are the process transfer functions, $u(t)$ is the control input and $e(t)$ is white noise with variance $\sigma_{e}^{2}$. Both transfer functions, $G_{0}(z)$ and $H_{0}(z)$, are rational and proper; furthermore, $H_{0}(\infty)=1$, that is the impulse response $h(t)$ of the filter $H_{0}(z)$ satisfies $h(0)=1$.

This true system may be under feedback control with a proper rational stabilizing controller $K(z)$ :

$$
u(t)=K(z)[r(t)-y(t)] .
$$

The system (1) is identified using a model structure parametrized by a vector $\theta \in \mathcal{R}^{d}$ :

$$
M(\theta): y(t)=G(z, \theta) u(t)+H(z, \theta) e(t) .
$$

It is assumed that the loop transfer function $G(z) K(z)$ has a non-zero delay, both for $G_{0}(z)$ and for all $G(z, \theta)$. The set of models $M(\theta)$, for all $\theta$ in some set $D_{\theta} \in \mathcal{R}^{d}$, defines the model set $\mathcal{M} \triangleq\left\{M(\theta) \mid \theta \in D_{\theta}\right\}$. The true system is said to belong to this model set, $\mathcal{S} \in \mathcal{M}$, if there is a $\theta_{0}$ such that $M\left(\theta_{0}\right)=\mathcal{S}$. In a prediction error identification framework, a model $[G(z, \theta) H(z, \theta)]$ uniquely defines the one-step-ahead predictor of $y(t)$ given all input/output data up to time $t$ :

$$
\hat{y}(t \mid t-1, \theta)=W_{u}(z, \theta) u(t)+W_{y}(z, \theta) y(t),
$$

where $W_{u}(z, \theta)$ and $W_{y}(z, \theta)$ are stable filters obtained from the model $[G(z, \theta) H(z, \theta)]$ as follows:

$W_{u}(z, \theta)=H^{-1}(z, \theta) G(z, \theta), W_{y}(z, \theta)=\left[1-H^{-1}(z, \theta)\right]$.

Since there is a $1-1$ correspondance between $[G(z, \theta), H(z, \theta)]$ and $\left[W_{u}(z, \theta), W_{y}(z, \theta)\right]$, the model $M(\theta)$ will in the future refer indistinctly to either one of these equivalent descriptions. For later use, we introduce the following vector notations:

$$
W(z, \theta) \triangleq\left[\begin{array}{ll}
W_{u}(z, \theta) & W_{y}(z, \theta)
\end{array}\right], \quad z(t) \triangleq\left[\begin{array}{l}
u(t) \\
y(t)
\end{array}\right]
$$

We shall also consider throughout this paper that the vector process $z(t)$ is quasistationary [6], so that the spectral density matrix $\Phi_{z}(\omega)$ is well defined.

The one-step-ahead prediction error is defined as:

$$
\begin{aligned}
\varepsilon(t, \theta) & \triangleq y(t)-\hat{y}(t \mid t-1, \theta)=y(t)-W(z, \theta) z(t) \\
& =H^{-1}(z, \theta)[y(t)-G(z, \theta) u(t)] .
\end{aligned}
$$

Using a set of $N$ input-output data and a least squares prediction error criterion yields the estimate $\hat{\theta}_{N}$ [6]:

$$
\hat{\theta}_{N}=\arg \min _{\theta \in D_{\theta}} \frac{1}{N} \sum_{t=1}^{N} \varepsilon^{2}(t, \theta) .
$$

Under reasonable conditions [6], $\hat{\theta}_{N} \stackrel{N \rightarrow \infty}{\longrightarrow} \theta^{*} \triangleq$ $\arg \min _{\theta \in D_{\theta}} \bar{V}(\theta)$, with

$$
\bar{V}(\theta) \triangleq \bar{E}\left[\varepsilon^{2}(t, \theta)\right]
$$

where

$$
\bar{E}[f(t)] \triangleq \lim _{N \rightarrow \infty} \frac{1}{N} \sum_{t=1}^{N} E[f(t)] .
$$

If $\mathcal{S} \in \mathcal{M}$ and if $\hat{\theta}_{N} \stackrel{N \rightarrow \infty}{\longrightarrow} \theta_{0}$, the parameter error converges to a Gaussian random variable:

$$
\sqrt{N}\left(\hat{\theta}_{N}-\theta_{0}\right) \stackrel{N \rightarrow \infty}{\longrightarrow} N\left(0, P_{\theta}\right)
$$

where

$$
\begin{aligned}
P_{\theta} & =\left.[I(\theta)]^{-1}\right|_{\theta=\theta_{0}}, \\
I(\theta) & =\frac{1}{\sigma_{e}^{2}} \bar{E}\left[\psi(t, \theta) \psi(t, \theta)^{T}\right], \\
\psi(t, \theta) & =-\frac{\partial \varepsilon(t, \theta)}{\partial \theta}=\frac{\partial \hat{y}(t \mid t-1, \theta)}{\partial \theta} \\
& =\nabla_{\theta} W(z, \theta) z(t),
\end{aligned}
$$

where $\nabla_{\theta} W(z, \theta) \triangleq \frac{\partial W(z, \theta)}{\partial \theta}$. The matrix $I\left(\theta_{0}\right)$ is called the information matrix. 


\section{IDENTIFIABILITY AND INFORMATIVE DATA}

Several concepts of identifiability have been proposed in the scientific literature, and these definitions have evolved over the years. Here we adopt a uniqueness-oriented definition proposed in [6], which deals with the injectivity of the mapping from parameter vector to model.

Definition 3.1: (Identifiability) A parametric model structure $M(\theta)$ is locally identifiable at a value $\theta_{1}$ if $\exists \delta>0$ such that, for all $\theta$ in $\left\|\theta-\theta_{1}\right\| \leq \delta$ :

$$
W(z, \theta)=W\left(z, \theta_{1}\right) \quad \forall z \Rightarrow \theta=\theta_{1} .
$$

The model structure is globally identifiable at $\theta_{1}$ if the same holds for $\delta \rightarrow \infty$. Finally, a model structure is globally identifiable if it is globally identifiable at almost all $\theta_{1}$.

The definition of identifiability (local, or global) is a property of the parametrization of the model $[G(z, \theta), \quad H(z, \theta)]$ or, equivalently, $\left[W_{u}(z, \theta), W_{y}(z, \theta)\right]$. It tells us that if the model structure is globally identifiable at some $\theta_{1}$, then there is no other parameter value $\theta \neq \theta_{1}$ that yields the exact same predictor as $M\left(\theta_{1}\right)$. However, it does not guarantee that the minimum, say $\theta_{1}$, of the asymptotic criterion $\bar{V}(\theta)$ is unique, even if the model structure is globally identifiable at $\theta_{1}$. This requires, additionally, that the data set is informative enough to distinguish between different predictors, which leads us to the definition of informative data with respect to a model structure.

Definition 3.2: (Informative data) [6] A quasistationary data set $z(t)$ is called informative with respect to a parametric model set $\left\{M(\theta), \theta \in \mathcal{D}_{\theta}\right\}$ if, for any two models $W\left(z, \theta_{1}\right)$ and $W\left(z, \theta_{2}\right)$ in that set,

$$
\bar{E}\left\{\left[W\left(z, \theta_{1}\right)-W\left(z, \theta_{2}\right)\right] z(t)\right\}^{2}=0
$$

implies

$$
W\left(e^{\jmath \omega}, \theta_{1}\right)=W\left(e^{\jmath \omega}, \theta_{2}\right) \quad \text { for almost all } \omega .
$$

By Parseval's theorem, we can rewrite:

$$
\begin{aligned}
& \bar{E}\left\{\left[W\left(z, \theta_{1}\right)-W\left(z, \theta_{2}\right)\right] z(t)\right\}^{2} \\
= & \frac{1}{2 \pi} \int_{-\pi}^{\pi}\left|W\left(e^{j \omega}, \theta_{1}\right)-W\left(e^{j \omega}, \theta_{2}\right)\right|^{2} \Phi_{z}(\omega) d \omega
\end{aligned}
$$

It is easy to see that an experiment that yields $\Phi_{z}(\omega)>0$ for almost all $\omega$ is informative for all model structures, but such condition is of course unnecessarily strong.

The definition of informative data is with respect to a given model set, not with respect to the true system, which may or may not belong to the model set. In an identification experiment, one typically first selects a globally identifiable model structure; this is a user's choice. Experimental conditions must then be selected that make the data informative with respect to that structure; this is again a user's choice. However, the data are generated by the true system, in open or in closed loop. Thus, the conditions that make a data set $z(t)$ informative with respect to some model structure depend on the true system and on the possible feedback configuration.

The main contribution of this paper will be to describe the weakest possible richness conditions on the input signal $u(t)$ (in open-loop identification) or $r(t)$ (in closed-loop identification) that make the data informative with respect to a given model structure. The degree of richness of a signal will be defined in the next section.

Finally, even though the results in this paper are not restricted to the situation where the true system is in the model set, in the situation where this is the case, we have the following classical result [6].

Proposition 3.1: Consider a model structure that can respresent the true system, i.e. $M\left(\theta_{0}\right)=\mathcal{S}$ for some $\theta_{0} \in \mathcal{D}_{\theta}$. Let this model structure be globally identifiable at $\theta_{0}$, and let the data be informative with respect to this model structure. Then $\theta_{0}$ is the unique global minimum of $\bar{V}(\theta)$ defined by (9), and in addition $I\left(\theta_{0}\right)>0$.

Convergence of an identification algorithm to the exact $\theta_{0}$ when $\mathcal{S} \in \mathcal{M}$ thus rests on the satisfaction of two different conditions: (i) the use of a model structure that is identifiable, at least at the global minimum $\theta_{0}$ of the asymptotic criterion $\bar{V}(\theta)$; (ii) the application of experiments that are informative with respect to the model structure used.

\section{RICH AND EXCITING SIGNALS}

In this section we introduce the concept of richness of a signal and of a persistently exciting regression vector. To motivate these definitions, we first derive explicit expressions for the pseudoregression vector $\psi(t, \theta)$ of (14) whose covariance defines the information matrix $I(\theta)$ through (13).

The pseudoregression vector can be written:

$$
\psi(t, \theta)=\left[\nabla_{\theta} W_{u}(z, \theta) \nabla_{\theta} W_{y}(z, \theta)\right]\left[\begin{array}{c}
u(t) \\
y(t)
\end{array}\right]
$$

We rewrite this gradient in terms of the external excitation signals, $u$ and $e$ in the case of open-loop data, $r$ and $e$ in the case of closed-loop data. We omit the explicit dependence on the variables $z$ and $\theta$ whenever it creates no confusion.

\section{Open-loop identification setup}

In open-loop identification, the data are generated as

$$
\left[\begin{array}{l}
u(t) \\
y(t)
\end{array}\right]=\left[\begin{array}{cc}
1 & 0 \\
G_{0} & H_{0}
\end{array}\right]\left[\begin{array}{l}
u(t) \\
e(t)
\end{array}\right]
$$

The pseudoregressor is then expressed in terms of the external signals as

$$
\begin{aligned}
\psi(t, \theta) & =\left[\begin{array}{ll}
\nabla_{\theta} W_{u}+\nabla_{\theta} W_{y} G_{0} & \nabla_{\theta} W_{y} H_{0}
\end{array}\right]\left[\begin{array}{l}
u(t) \\
e(t)
\end{array}\right] \\
& \triangleq V_{u o l}(z, \theta) u(t)+V_{\text {eol }}(z, \theta) e(t)
\end{aligned}
$$

\section{Closed-loop identification setup}

In closed-loop identification, the data are generated as

$$
\left[\begin{array}{l}
u(t) \\
y(t)
\end{array}\right]=S\left[\begin{array}{cc}
K & -K H_{0} \\
K G_{0} & H_{0}
\end{array}\right]\left[\begin{array}{l}
r(t) \\
e(t)
\end{array}\right]
$$


where $K=K(z)$ is the controller, and $S=S(z)=$ $\frac{1}{1+K(z) G_{0}(z)}$ is the sensitivity function. The pseudoregressor is then expressed in terms of the external signals as

$$
\begin{aligned}
& \psi(t, \theta) \\
& =\left[S K\left(\nabla_{\theta} W_{u}+\nabla_{\theta} W_{y} G_{0}\right)\right. \\
& \left.\quad S H_{0}\left(\nabla_{\theta} W_{y}-K \nabla_{\theta} W_{u}\right)\right] \\
& \times\left[\begin{array}{c}
r(t) \\
e(t)
\end{array}\right] \\
& \triangleq V_{r c l}(z, \theta) r(t)+V_{e c l}(z, \theta) e(t)
\end{aligned}
$$

We observe that in both setups the pseudoregressor is a sum of two $d$-vectors of quasistationary signals: the first is obtained as the output of a $d$-vector of proper stable rational transfer functions driven by the known scalar external signal ( $u$ or $r$ ); the second is obtained as the output of a $d$-vector of proper stable rational transfer functions driven by the white noise signal $e$. We now introduce the following classical definitions.

Definition 4.1: A quasistationary vector signal $\psi(t)$ is called persistently exciting (PE) if $\bar{E}\left[\psi(t) \psi^{T}(t)\right]>0$.

Whether a quasistationary vector signal $\psi(t)$ obtained as a filtered version (by a vector $V(z)$ of transfer functions) of a quasistationary scalar signal $u(t)$ is $\mathrm{PE}$ or not depends not only on $V(z)$ but also on the degree of richness of the input $u(t)$. The richness of a scalar signal is defined as follows.

Definition 4.2: A quasistationary scalar signal $u(t)$ is sufficiently rich of order $n$ (denoted $\mathrm{SRn}$ ) if the following regressor is $\mathrm{PE}$ :

$$
\phi_{1, n}(t) \triangleq\left[\begin{array}{c}
u(t-1) \\
u(t-2) \\
\vdots \\
u(t-n)
\end{array}\right]=\left[\begin{array}{c}
z^{-1} \\
z^{-2} \\
\vdots \\
z^{-n}
\end{array}\right] u(t)
$$

The vector $\phi_{1, n}(t)$ serves as a basis for all regression vectors that are obtained as (vector)-filtered versions of a scalar signal $u(t)$. For future use, we introduce the notation:

$$
\mathcal{B}_{k, n}(z) \triangleq\left[\begin{array}{llll}
z^{-k} & z^{-k-1} & \ldots & z^{-n}
\end{array}\right]^{T}, \text { for } k \leq n .
$$

Observe that, by our assumption of quasistationarity, $u(t)$ is $\mathrm{SRn}$ if $\mathcal{B}_{k+1, k+n}(z) u(t)$ is $\mathrm{PE}$ for any $k$. Thus, we could just as well have used $\phi_{0, n-1}(t)$ in lieu of $\phi_{1, n}(t)$ in Definition 4.2: the definition is shift-invariant. We denote by $\mathcal{U}_{n}$ the set of all $\mathbf{S R n}$ signals.

Proposition 4.1: A scalar quasistationary signal $u(t)$ is SRn if

- its spectral density is nonzero in at least $n$ frequency points in the interval $(-\pi, \pi]$.

- it cannot be filtered to zero by a FIR filter of degree $n-1$ : $\alpha_{1} z^{-1}+\ldots+\alpha_{n} z^{-n}$.

A scalar signal $u(t)$ is SREn if its spectral density is nonzero in exactly $n$ frequency points in the interval $(-\pi, \pi]$.

The equivalence comes by observing that

$$
\begin{aligned}
& \alpha^{T} \bar{E}\left[\phi_{1, n}(t) \phi_{1, n}^{T}(t)\right] \alpha \\
& =\frac{1}{2 \pi} \int_{-\pi}^{\pi}\left|\alpha_{1} e^{-j \omega}+\ldots+\alpha_{n} e^{-j n \omega}\right|^{2} \Phi_{u}(\omega) d \omega .
\end{aligned}
$$

\section{INFORMATIVITY OF THE DATA SET FOR ARMAX AND BJ MODEL STRUCTURES}

In this section we derive necessary and sufficient conditions for the informativity of the data set for ARMAX and BJ model structures, as well as for the special cases of ARX and OE model structures.

\section{A. Open-loop identification}

Recall Definition 3.2 for informative data. We introduce the following shorthand notation (see (6)):

$$
\begin{aligned}
& \triangle W_{u} \triangleq W_{u}\left(z, \theta_{1}\right)-W_{u}\left(z, \theta_{2}\right) \\
& \triangle W_{y} \triangleq W_{y}\left(z, \theta_{1}\right)-W_{y}\left(z, \theta_{2}\right) .
\end{aligned}
$$

For open loop data, it follows from (19) that:

$$
\begin{aligned}
& {\left[W\left(z, \theta_{1}\right)-W\left(z, \theta_{2}\right)\right] z(t)} \\
& =\left[\triangle W_{u}+\triangle W_{y} G_{0}\right] u(t)+\triangle W_{y} H_{0} e(t) .
\end{aligned}
$$

Given the independence between $u$ and $e$, condition (15) is therefore equivalent with the following set of conditions:

$$
\begin{aligned}
& \bar{E}\left\{\left[\triangle W_{u}+\triangle W_{y} G_{0}\right] u(t)\right\}^{2}=0 \\
& \bar{E}\left\{\triangle W_{y} H_{0} e(t)\right\}^{2}=0
\end{aligned}
$$

We now seek the smallest degree of richness of $u$ for which the conditions (27)-(28) imply $\triangle W_{u} \equiv 0$ and $\triangle W_{y} \equiv 0$. Since (28) implies $\triangle W_{y} \equiv 0$, this is equivalent with finding necessary and sufficient conditions on the richness of $u$ such that

$$
E\left\{\left[\triangle W_{u}\right] u(t)\right\}^{2}=0 \Longrightarrow \triangle W_{u} \equiv 0
$$

\section{ARMAX model structure}

Consider first the ARMAX model structure

$$
A\left(z^{-1}\right) y(t)=B\left(z^{-1}\right) u(t)+C\left(z^{-1}\right) e(t)
$$

where $A\left(z^{-1}\right)=1+a_{1} z^{-1}+\ldots+a_{n_{a}} z^{-n_{a}}$, $B\left(z^{-1}\right)=b_{1} z^{-1}+\ldots+b_{n_{b}} z^{-n_{b}}$, and $C\left(z^{-1}\right)=$ $1+c_{1} z^{-1}+\ldots+c_{n_{c}} z^{-n_{c}}$. We comment that for ARMAX model structures, one must consider as generic the possible existence of common roots between the polynomials $A$ and $B$, as well as between $A$ and $C$. However, the three polynomials $A, B$ and $C$ must be coprime at any identifiable $\theta$. We then have the following result.

Theorem 5.1: For the ARMAX model structure (30), the data set is informative if and only if $u(t)$ is SRk, where $k=n_{b}+\min \left\{n_{a}, n_{c}\right\}$.

Proof: Since $\triangle W_{y} \equiv 0$ it follows that

$$
\frac{C\left(z, \theta_{1}\right)}{A\left(z, \theta_{1}\right)}=\frac{C\left(z, \theta_{2}\right)}{A\left(z, \theta_{2}\right)}
$$

for any pair of values $\theta_{1}$ and $\theta_{2}$. Since the polynomials $A$ and $C$ may have common roots, let $U(z)$ be the greatest common factor of $A\left(z, \theta_{1}\right)$ and $C\left(z, \theta_{1}\right)$ :

$$
A\left(z, \theta_{1}\right)=A_{1}\left(z, \theta_{1}\right) U(z), \quad C\left(z, \theta_{1}\right)=C_{1}\left(z, \theta_{1}\right) U(z),
$$


with $U(z)=1+u_{1} z^{-1}+\ldots+u_{n_{u}} z^{-n_{u}}$. Then $\triangle W_{y} \equiv 0$ is equivalent with

$$
A\left(z, \theta_{2}\right) C_{1}\left(z, \theta_{1}\right)-C\left(z, \theta_{2}\right) A_{1}\left(z, \theta_{1}\right)=0
$$

with $A_{1}\left(z, \theta_{1}\right)$ and $C_{1}\left(z, \theta_{1}\right)$ coprime. The general solution of (31) is

$$
A\left(z, \theta_{2}\right)=A_{1}\left(z, \theta_{1}\right) T(z), \quad C\left(z, \theta_{2}\right)=C_{1}\left(z, \theta_{1}\right) T(z),
$$

where $T(z)$ is an arbitrary monic polynomial of the same degree as $U$. For this ARMAX model structure, the left hand side of (29), expressed at those two values $\theta_{1}$ and $\theta_{2}$, is equivalent with:

$$
\bar{E}\left\{\left[A\left(z, \theta_{2}\right) B\left(z, \theta_{1}\right)-A\left(z, \theta_{1}\right) B\left(z, \theta_{2}\right)\right] u(t)\right\}^{2}=0,
$$

which is equivalent with

$$
\bar{E}\left\{\left[T(z) B\left(z, \theta_{1}\right)-U(z) B\left(z, \theta_{2}\right)\right] u(t)\right\}^{2}=0 .
$$

This implies

$$
T(z) B\left(z, \theta_{1}\right)-U(z) B\left(z, \theta_{2}\right) \equiv 0
$$

if and only if $u(t) \in \mathcal{U}_{n_{b}+n_{u}}$. Note that $U(z)$ and $B\left(z, \theta_{1}\right)$ are coprime by definition of $U(z)$. The general solution of (34) is therefore

$$
B\left(z, \theta_{2}\right)=M(z) B\left(z, \theta_{1}\right), \quad T(z)=M(z) U(z) .
$$

However, since $T(z)$ and $U(z)$ have the same degree, $n_{u}$, and since both are monic, the only solution is $M(z)=1$, which implies that $\theta_{1}=\theta_{2}$. We conclude that the predictor at $\theta_{1}$ is identical to the predictor at any other value $\theta_{2}$ if and only if $u(t) \in \mathcal{U}_{n_{b}+n_{u}}$ where $n_{u}$ is the number of common roots between $A(z, \theta)$ and $C(z, \theta)$ at $\theta_{1}$. For the data to be informative with respect to the ARMAX model structure, this must hold at all values of $\theta_{1}$. The stated result then follows, since the maximum number of common roots between $A(z, \theta)$ and $C(z, \theta)$ is $\min \left\{n_{a}, n_{c}\right\}$.

The corresponding result for an ARX model structure follows immediately.

Corollary 5.1: For the ARX model structure $A\left(z^{-1}\right) y(t)$ $=B\left(z^{-1}\right) u(t)+e(t)$ with $A$ and $B$ as above, the data set is informative if and only if $u(t) \in \mathcal{U}_{n_{b}}$.

\section{BJ model structure}

Consider now the BJ model structure:

$$
y(t)=\frac{B\left(z^{-1}\right)}{F\left(z^{-1}\right)} u(t)+\frac{C\left(z^{-1}\right)}{D\left(z^{-1}\right)} e(t)
$$

where $B\left(z^{-1}\right)$ and $C\left(z^{-1}\right)$ are as above, with $F\left(z^{-1}\right)=1+f_{1} z^{-1}+\ldots+f_{n_{f}} z^{-n_{f}}$ and $D\left(z^{-1}\right)=1+d_{1} z^{-1}+\ldots+d_{n_{d}} z^{-n_{d}}$. We have the following result.

Theorem 5.2: For the BJ model structure (35) operating in open loop, the data are informative if and only if $u(t)$ is SRk, where $k=n_{b}+n_{f}$.
Proof: It follows from (31) that $\frac{C\left(z, \theta_{1}\right)}{D\left(z, \theta_{1}\right)}=\frac{C\left(z, \theta_{2}\right)}{D\left(z, \theta_{2}\right)}$. Therefore, (32) is equivalent with $\bar{E}\left\{\frac{D\left(z, \theta_{1}\right)}{C\left(z, \theta_{1}\right)}\left[\frac{B\left(z, \theta_{1}\right)}{F\left(z, \theta_{1}\right)}-\right.\right.$ $\left.\left.\frac{B\left(z, \theta_{2}\right)}{F\left(z, \theta_{2}\right)}\right] u(t)\right\}^{2}=0$, or equivalently,

$$
\bar{E}\left\{\left[F\left(z, \theta_{2}\right) B\left(z, \theta_{1}\right)-F\left(z, \theta_{1}\right) B\left(z, \theta_{2}\right)\right] u(t)\right\}^{2}=0 .
$$

Since the degree of the polynomial that filters $u(t)$ in (36) is $n_{b}+n_{f}$, the result then follows immediately.

Corollary 5.2: For the OE model structure $y(t)=$ $\frac{B\left(z^{-1}\right)}{F\left(z^{-1}\right)} u(t)+e(t)$, the richness condition on $u(t)$ is identical to that for the BJ model structure.

\section{$B$. Closed-loop identification}

For closed-loop data, it follows from (21) that

$$
\begin{aligned}
& {\left[W\left(z, \theta_{1}\right)-W\left(z, \theta_{2}\right)\right] z(t)=} \\
& K S\left[\triangle W_{u}+\triangle W_{y} G_{0}\right] r(t)+H_{0} S\left[\triangle W_{y}-K \triangle W_{u}\right] e(t)
\end{aligned}
$$

Given the independence between $r$ and $e$, condition (15) is therefore equivalent with the following set of conditions:

$$
\begin{aligned}
& \bar{E}\left\{K S\left[\triangle W_{u}+\triangle W_{y} G_{0}\right] r(t)\right\}^{2}=0 \\
& \bar{E}\left\{H_{0} S\left[\triangle W_{y}-K \triangle W_{u}\right] e(t)\right\}^{2}=0
\end{aligned}
$$

These conditions, in turn, are equivalent with the following set of conditions

$$
\begin{aligned}
& \triangle W_{y} \equiv K \triangle W_{u}, \\
& \bar{E}\left\{K \triangle W_{u} r(t)\right\}^{2}=0,
\end{aligned}
$$

where the second expression follows by substituting the first in (38) and using $S=\left(1+K G_{0}\right)^{-1}$. Note that, given the first condition, the second is equivalent with

$$
\bar{E}\left\{\triangle W_{y} r(t)\right\}^{2}=0
$$

For the controller $K(z)$ of (2) we shall consider a coprime factorization $K(z)=\frac{X\left(z^{-1}\right)}{Y\left(z^{-1}\right)}$, with $X\left(z^{-1}\right)=x_{0}+x_{1} z^{-1}+\ldots+x_{n_{x}} z^{-n_{x}}$ and $Y\left(z^{-1}\right)=1+y_{1} z^{-1}+\ldots+y_{n_{y}} z^{-n_{y}}$.

\section{ARMAX model structure}

We first consider the ARMAX model structure (30) under feedback control with the stabilizing controller $K(z)=\frac{X\left(z^{-1}\right)}{Y\left(z^{-1}\right)}$. For simplicity, we shall consider only parameter values $\theta$ at which the following assumption holds.

Assumption 1: The polynomials $A\left(z^{-1}\right) Y\left(z^{-1}\right)+$ $B\left(z^{-1}\right) X\left(z^{-1}\right)$ and $C\left(z^{-1}\right)$ are coprime.

Notice that the subset of $\theta$ values at which these polynomials have a common root has measure zero in the parameter space. They correspond to parameter values that cause a polezero cancellation between the closed-loop poles of the model and the zeros of the noise model. We then have the following result.

Theorem 5.3: Consider the ARMAX model structure (30) under feedback control with the stabilizing controller 
$K(z)=\frac{X\left(z^{-1}\right)}{Y\left(z^{-1}\right)}$, with Assumption 1 holding.

(i) Let $r(t) \equiv 0$. Then the data are informative if and only if

$$
\max \left(n_{x}-n_{a}, n_{y}-n_{b}\right) \geq 0 .
$$

(ii) Let $\max \left(n_{x}-n_{a}, n_{y}-n_{b}\right)<0$. Then the data are informative for almost all $r(t) \in \mathcal{U}_{k}$ if and only if

$$
k \geq \min \left(n_{a}-n_{x}, n_{b}-n_{y}\right) .
$$

Proof: For the ARMAX model structure (30), the identity (40) is equivalent with ${ }^{1}$

$$
\left(A_{2} Y+B_{2} X\right) C_{1}=\left(A_{1} Y+B_{1} X\right) C_{2},
$$

where the pairs of polynomials $C_{1}$ and $A_{1} Y+B_{1} X$, as well as $C_{2}$ and $A_{2} Y+B_{2} X$ are coprime by Assumption 1. Since $C_{1}$ and $A_{1} Y+B_{1} X$ are coprime, the general solution of (45) is

$$
A_{2} Y+B_{2} X=M\left(A_{1} Y+B_{1} X\right), \quad C_{2}=M C_{1},
$$

where $M(z)$ is an arbitrary polynomial. But since $C_{1}(z)$ and $C_{2}(z)$ are monic with the same degree, the only solution is $M(z)=1$, i.e.

$$
C_{1}=C_{2} \text { and }\left(A_{1}-A_{2}\right) Y+\left(B_{1}-B_{2}\right) X=0 .
$$

Since $X$ and $Y$ are coprime, the general solution of this last equality is given by

$$
\triangle A=T X, \triangle B=-T Y,
$$

where $T(z)=t_{1} z^{-1}+\ldots+t_{n_{t}} z^{-n_{t}}$ is again an arbitrary polynomial with $\operatorname{deg} T(z)=n_{t} \triangleq \min \left\{n_{a}-n_{x}, n_{b}-n_{y}\right\}$. $T(z)=0$ is the only solution of (48), and hence the data are informative without any external excitation, if and only condition (43) holds.

Now consider the case where $\max \left(n_{x}-n_{a}, n_{y}-n_{b}\right)<$ 0 , i.e. $\min \left\{n_{a}-n_{x}, n_{b}-n_{y}\right\} \geq 1$. It then follows from $C_{1}=C_{2}$ and $\triangle B=-T Y$ that condition (41) is equivalent with $\bar{E}\{X \operatorname{Tr}(t)\}^{2}=0$. This implies $T(z) \equiv 0$, and hence the data are informative, if and only if $r(t) \in \mathfrak{U}_{k}$ with $k \geq$ $\min \left(n_{a}-n_{x}, n_{b}-n_{y}\right)$ provided the points of support of $r(t)$ do not coincide with possible zeroes of $X$ on the unit circle. This proves part (ii) of the Theorem.

Comment. An ARMAX model identified in closed loop is identifiable from noise information only if the controller is sufficiently complex with respect to the model structure, in a way that is made precise by the condition (43); this condition is known and can be found in [8]. What is novel and, we believe, remarkable in Theorem 5.3 is that, when that complexity condition is not satisfied by the controller, then the degree of richness required of the reference signal is precisely determined by how much that condition is violated. In other words, the degree of richness required of $r(t)$ is precisely equal to the difference between the complexity required by expression (43) and the actual complexity of the controller.

\footnotetext{
${ }^{1}$ To keep notations simple, we drop the $z$ argument here.
}

Corollary 5.3: For the ARX model structure $A\left(z^{-1}\right) y(t)=B\left(z^{-1}\right) u(t)+e(t)$ under feedback control with the stabilizing controller $K(z)=\frac{X\left(z^{-1}\right)}{Y\left(z^{-1}\right)}$, the richness conditions are identical to those given in Theorem 5.3 for the ARMAX model structure.

Proof: The proof follows immediately by setting $C\left(z^{-1}\right)=1$ everywhere in the proof of Theorem 5.3.

\section{BJ model structure}

We now consider the BJ model structure (35) under feedback control with the stabilizing controller $K(z)=\frac{X\left(z^{-1}\right)}{Y\left(z^{-1}\right)}$. For simplicity, we shall again exclude parameter values $\theta$ that cause a pole-zero cancellation between the closed-loop poles of the model and the zeros of the noise model. This corresponds to the following assumption.

Assumption 2: The polynomials $F\left(z^{-1}\right) Y\left(z^{-1}\right)+$ $B\left(z^{-1}\right) X\left(z^{-1}\right)$ and $C\left(z^{-1}\right)$ are coprime.

We then have the following result.

Theorem 5.4: Consider the BJ model structure (35) under feedback control with the stabilizing controller $K(z)=$ $\frac{X\left(z^{-1}\right)}{Y\left(z^{-1}\right)}$, with Assumption 2 holding.

(i) Let $r(t) \equiv 0$. Then the data are informative if and only if

$$
\max \left(n_{x}-n_{f}, n_{y}-n_{b}\right) \geq n_{d}+\min \left\{n_{x}, n_{f}\right\} .
$$

(ii) Let $\max \left(n_{x}-n_{f}, n_{y}-n_{b}\right)<n_{d}+\min \left\{n_{x}, n_{f}\right\}$. Then the data are informative for almost all $r(t) \in \mathcal{U}_{k}$ if and only if

$k \geq n_{d}+\min \left\{n_{x}, n_{f}\right\}+\min \left(n_{f}-n_{x}, n_{b}-n_{y}\right)$

Proof: For the BJ model structure, the identity (40) is equivalent with

$$
\frac{\left(F_{1} Y+B_{1} X\right) D_{1}}{C_{1} F_{1}}=\frac{\left(F_{2} Y+B_{2} X\right) D_{2}}{C_{2} F_{2}} .
$$

Suppose first that $D_{1}$ and $F_{1}$ have a common polynomial factor $H(z)=1+h_{1} z^{-1}+\ldots+h_{n_{h}} z^{-n_{h}}$, so that $D_{1}=$ $\bar{D}_{1} H$ and $F_{1}=\bar{F}_{1} H$, with $\bar{D}_{1}$ and $\bar{F}_{1}$ coprime. Consider, additionally, that there are possible pole-zero cancellations at $\theta_{1}$ between the zeroes of the controller and the poles of $G\left(\theta_{1}\right)$, i.e. let $M(z)=1+m_{1} z^{-1}+\ldots+m_{n_{m}} z^{-n_{m}}$ be the greatest common factor between $X$ and $\bar{F}_{1}$, so that $X=$ $X_{1} M$ and $\bar{F}_{1}=F_{1}^{*} M$ with $X_{1}$ and $F_{1}^{*}$ coprime. Note that $F_{1}=F_{1}^{*} M H$. Then (51) is equivalent with

$$
\frac{\left(F_{1}^{*} H Y+B_{1} X_{1}\right) \bar{D}_{1}}{C_{1} F_{1}^{*}}=\frac{\left(F_{2} Y+B_{2} X\right) D_{2}}{C_{2} F_{2}},
$$

where $C_{1} F_{1}^{*}$ and $\left(F_{1}^{*} H Y+B_{1} X_{1}\right) \bar{D}_{1}$ are now coprime because $C_{1}$ is coprime with $D_{1}$ (and hence with $\bar{D}_{1}$ ) and also with $F_{1} Y+B_{1} X$ (and hence with $F_{1}^{*} H Y+B_{1} X_{1}$ ) by Assumption 1. In addition, $F_{1}^{*}$ is coprime with $B_{1}, \bar{D}_{1}$ and $X_{1}$, and hence with the whole numerator. The general solution is therefore

$$
\begin{aligned}
C_{2} F_{2} & =C_{1} F_{1}^{*} T \\
\left(F_{2} Y+B_{2} X\right) D_{2} & =\left(F_{1}^{*} H Y+B_{1} X_{1}\right) \bar{D}_{1} T
\end{aligned}
$$


where $T=1+t_{1} z^{-1}+\ldots+t_{n_{t}} z^{-n_{t}}$ of degree $n_{t}=n_{m}+n_{h}$. Equation (54) can be rewritten as

$$
\left(F_{2} D_{2}-F_{1}^{*} H \bar{D}_{1} T\right) Y+\left(B_{2} D_{2} M-B_{1} \bar{D}_{1} T\right) X_{1}=0
$$

with $X_{1}$ and $Y$ coprime. Note that the leading term of the polynomials multiplying $Y$ and $X_{1}$ is zero. The general solution of (55) is given by

$$
\begin{aligned}
F_{2} D_{2}-F_{1}^{*} H \bar{D}_{1} T & =X_{1} U \\
B_{2} D_{2} M-B_{1} \bar{D}_{1} T & =-Y U
\end{aligned}
$$

where $U$ is an arbitrary polynomial of the form $U(z)=$ $u_{1} z^{-1}+\ldots+u_{n_{u}} z^{-n_{u}}$, with $n_{u}=n_{d}+n_{m}+\min \left\{n_{f}-\right.$ $\left.n_{x}, n_{b}-n_{y}\right\} . U(z)=0$ is the only solution of (56)-(57) if and only if $\max \left\{n_{x}-n_{f}, n_{y}-n_{b}\right\} \geq n_{d}+n_{m}$. Suppose this is the case; it then follows from (56)-(57) that

$$
\begin{aligned}
F_{2} D_{2} & =F_{1}^{*} H \bar{D}_{1} T \text { and } \\
B_{2} D_{2} M & =B_{1} \bar{D}_{1} T
\end{aligned}
$$

Combining (53) and (58) yields

$$
\frac{C_{2}}{D_{2}}=\frac{C_{1}}{H \bar{D}_{1}}=\frac{C_{1}}{D_{1}},
$$

while combining (58) and (59) yields

$$
\frac{B_{2}}{F_{2}}=\frac{B_{1}}{F_{1}^{*} H M}=\frac{B_{1}}{F_{1}} .
$$

Together, these last two identities imply that the data are sufficiently informative to distinguish between the model at $\theta_{1}$ and any other model. Part (i) of the Theorem then follows from the fact that $n_{m}$ is the number of common roots between the zeroes of the controller and the poles of the plant model and that the largest possible number of such common roots, for any value of $\theta_{1}$, is $\min \left\{n_{x}, n_{f}\right\}$.

Consider now the case where the controller is not sufficiently complex to produce informative data with noise excitation only, i.e. condition (49) is violated. We then seek necessary and sufficient richness conditions on the external excitation $r(t)$ under which conditions (40) and (41) or, equivalently (42), imply $\triangle W_{u} \equiv 0$ and $\triangle W_{y} \equiv 0$. It follows from the previous derivations that

$$
\begin{aligned}
\triangle W_{y} & =\frac{C_{1} D_{2}-D_{1} C_{2}}{C_{1} C_{2}} \\
& =\frac{1}{C_{1} C_{2}}\left[C_{1} D_{2}-D_{1} \frac{C_{1} F_{1}^{*} T}{F_{2}}\right] \\
& =\frac{1}{F_{2} C_{2}}\left[D_{2} F_{2}-D_{1} F_{1}^{*} T\right]=\frac{X_{1} U}{F_{2} C_{2}}
\end{aligned}
$$

Thus, (42) implies $\triangle W_{y} \equiv 0$ if and only if $E\left\{\frac{X_{1} U}{F_{2} C_{2}} r(t)\right\}^{2}=$ 0 implies $U(z) \equiv 0$. Remember that $\operatorname{deg}(U) \triangleq n_{u}=n_{d}+$ $n_{m}+\min \left\{n_{f}-n_{x}, n_{b}-n_{y}\right\}$ where $n_{m}$ is the number of common roots between the controller zeroes and the poles of $G(z, \theta)$ at the considered $\theta_{1}$. Since the largest number of such pole-zero cancellations at any $\theta_{1}$ is $\min \left\{n_{x}, n_{f}\right\}$, it then follows that $U(z) \equiv 0$ if and only if $r(t) \in \mathcal{U}_{k}$ for all $k \geq n_{d}+\min \left\{n_{x}, n_{f}\right\}+\min \left(n_{f}-n_{x}, n_{b}-n_{y}\right)$ provided the points of support of $u(t)$ do not coincide with possible zeroes of $X$ on the unit circle. This proves part (ii) of the Theorem.

Comment. We observe that, just like in the case of an ARMAX model structure identified in closed loop, the degree of richness required of the external excitation signal $r(t)$ is precisely equal to the difference between the complexity required by expression (49) and the actual complexity of the controller.

Corollary 5.4: For the OE model structure $y(t)=$ $\frac{B\left(z^{-1}\right)}{F\left(z^{-1}\right)} u(t)+e(t)$, under feedback control with the stabilizing controller $K(z)=\frac{X\left(z^{-1}\right)}{Y\left(z^{-1}\right)}$, the data set is informative if and only if $K(z) \neq 0$.

Proof: Since $W_{y}(z)=0$ for an OE model, condition (39) is equivalent with $K \triangle W_{u} \equiv 0$. Since $W_{u}=\frac{B_{1}}{F_{1}}-\frac{B_{2}}{F_{2}}$ this implies $\frac{B_{1}}{F_{1}}=\frac{B_{2}}{F_{2}}$ if and only if $K(z) \neq 0$.

This confirms a result obtained in [3] where it was shown that an OE model structure is identifiable in closed loop without external excitation if the controller is not identically zero.

\section{CONCLUSIONS}

We have provided necessary and sufficient conditions on the external signals to achieve informative data for all commonly used input-output models, under both open-loop and closed-loop experimental conditions. Our objective has been to find the smallest possible degree of richness of the external signal ( $u$ in open loop, $r$ in closed loop) that delivers an informative data set. While the open-loop results were either known or to be expected, the novel contribution of this paper lies with the closed-loop results. They show a remarkable and precisely quantifiable trade-off between the controller complexity and the required degree of external excitation. Our present work consists in establishing the tightest possible connection between informativity of the data set and positivity of the information matrix.

\section{REFERENCES}

[1] A.S. Bazanella, M. Gevers, and L. Mišković. Closed-loop identification of MIMO systems: a new look at identifiability and experiment design. In CD-ROM Proc. of European Control Conference, pages 5694-5699, Kos, Greece, July 2007.

[2] X. Bombois, G. Scorletti, M. Gevers, R. Hildebrand, and P.M.J. Van den Hof. Cheapest open-loop identification for control. In CD-ROM Proc. 33rd IEEE Conf on Decision and Control, pages 382-387, The Bahamas, December 2004.

[3] X. Bombois, G. Scorletti, M. Gevers, P.M.J. Van den Hof, and R. Hildebrand. Least costly identification experiment for control. Automatica, 42(10):1651-1662, October 2006.

[4] M. Gevers, L. Mišković, D. Bonvin, and A. Karimi. Identification of multi-input systems: variance analysis and input design issues. Automatica, 42(4):559-572, April 2006.

[5] H. Jansson and H. Hjalmarsson. Optimal experiment design in closed loop. In 16th IFAC World Congress on Automatic Control, paper 04528, July 2005.

[6] L. Ljung. System Identification: Theory for the User, 2nd Edition. Prentice-Hall, Englewood Cliffs, NJ, 1999.

[7] T. S. Ng, G. C. Goodwin, and B. D. O. Anderson. Identifiability of MIMO linear dynamic systems operating in closed loop. Automatica, 13:477-485, 1977.

[8] T. Söderström and P. Stoica. System Identification. Prentice-Hall International, Hemel Hempstead, Hertfordshire, 1989. 